\title{
PREDICCIÓN METEOROLÓGICA ESTACIONAL Y SU COMUNICACIÓN AL PEQUEÑO AGRICULTOR EN EL NORDESTE DE BRASIL
}

\author{
Manuel Cabalar Fuentes \\ Departamento de Ciências Humanas e Filosofía \\ Universidade Estadual de Feira de Santana (UEFS). Bahia-Brasil
}

\section{RESUMEN}

Muchos aspectos de la vida cotidiana de las personas son influenciadas por las condiciones meteorológicas. Este hecho es más claro si la actividad se desarrolla al aire libre, expuesta directamente a los avatares atmosféricos. Bien conocido es el caso de la agricultura y la ganadería, que para tener éxito y rendir adecuadamente necesitan de agua y temperaturas dentro de unos determinados umbrales. En este contexto, las predicciones meteorológicas importan mucho, pues ayudan a los productores a planificar mejor sus actividades, anticiparse a circunstanciales eventos negativos y mejorar, por tanto, las cosechas. Son más vulnerables los pequeños agricultores familiares, sin medios técnicos y con poco o ningún acceso a la información meteorológica, y es peor aún en áreas de Clima irregular como las semiáridas, donde las lluvias caen erráticamente y de vez en cuando las sequías causan grandes estragos precisamente en esos pequeños productores. En este trabajo vamos a hacer una aproximación teórica a la problemática de la comunicación de las predicciones meteorológicas a pequeños agricultores, con atención especial en el Sertão (el espacio semiárido del Nordeste de Brasil), completado con una visión previa de los mecanismos atmosféricos y oceánicos que influencian su clima, bien conocidos y monitoreados continuamente, facilitando la predicción anticipada de las condiciones meteorológicas estacionales.

Palabras clave: predicción meteorológica, pequeño agricultor, comunicación, semiárido, Sertão, Brasil.

\section{ABSTRACT}

\section{Seasonal forecast and its communication to small farmer in Northeast Brazil}

Many aspects of people's daily lives are influenced by the weather. This fact become more apparent when it involves outdoor activities, directly exposed to the atmospheric variability. Well known is the case of agriculture and livestock, where it is crucial, within certain thresholds, to perform adequately the need of water and temperature. In this context, the weather forecasts is considered significant, as this helps farmers to plan their activities, anticipate negative events and thereby improving the crop yields.

Usually, small family farmers are the most vulnerable group without technical resources and with low or no access to weather information. Particularly, territories with semi-arid climate, where the rainfalls are erratically and occasionally it can lead to droughts, represent the worse scenario for small producers.

In this paper we make a theoretical approach to the problem of communication of weather forecasts to small farmers, with special focus on Sertão (the semi-arid territory in the Northeast of Brazil). For understanding the case study, we complete this article with a previous summary of the atmospheric and oceanic mechanisms that influence their climate, well-known and continuously monitored, providing early prediction of seasonal weather conditions.

Keywords: seasonal forecast, small farmer, communication, semi-arid Sertão, Brazil.

\section{INTRODUCCIÓN: LAS REGIONES SEMIÁRIDAS COMO EJEMPLO DEL IMPACTO DE LOS EXTREMOS CLIMÁTICOS EN EL MUNDO}

A pesar de los avances tecnológicos que la sociedad humana ha experimentado en el último siglo, hasta ahora no ha sido capaz de alcanzar la total invulnerabilidad a las manifestaciones más extremas de

Contacto: manuel.cabalar@gmail.com 
las fuerzas naturales. Terremotos, erupciones volcánicas, tempestades y sequías son eventos naturales que la ciencia nos ha permitido entender y explicar con gran detalle desde su génesis al seguimiento de todos sus estadios de desarrollo, pero no nos ha proporcionado medios para quedar totalmente indemnes a sus efectos. En la mejor de las hipótesis, ha permitido anticipar su ocurrencia y en consecuencia reducir sus impactos. En realidad, no es un atrevimiento decir que hoy en día, los seres humanos son más vulnerables que nunca a los desastres naturales. Con frecuencia los medios de comunicación nos muestran los impactos de numerosas catástrofes alrededor del Mundo, costando la vida de muchas personas y causando importantes daños materiales. El crecimiento continuo de la población, que se ha extendido por lugares remotos y peligrosos antes deshabitados, y como consecuencia directa de este proceso, la instalación de sus hogares, actividades cotidianas e infraestructuras en áreas inadecuadas ha aumentado en alto grado la exposición a los mayores impactos de la naturaleza (esencia del concepto de "Riesgo Natural") y las catástrofes, consecuentemente, han aumentado su frecuencia y magnitud.

Entre los eventos naturales, la acción del Clima (o más exactamente, los eventos climáticos extremos) han alcanzado una posición particularmente importante en los foros de discusión popular e intelectual. Hace mucho tiempo que se discute sobre la variabilidad climática y el aumento de la nitidez y la frecuencia de los extremos (calor y frio intensos, lluvias torrenciales y sequías largas), que son casi siempre asociados al llamado "Cambio Climático" supuestamente inducido por las actividades humanas. No entra en los objetivos de este trabajo contribuir a este debate, ni indagar si el "Cambio Climático" es real, porque el Clima está siempre cambiando a diversas escalas temporales, ni tampoco discutir si el cambio es producido por el ser humano exclusivamente o también influyen factores naturales.

En cualquier caso, más allá de estas disquisiciones, no existe duda de que hay ciertos lugares en el mundo que, por su posición en el Globo, son frecuentemente influenciados por eventos climáticos extremos, y cuyas poblaciones conviven con ellos desde siempre con mayor o menor fortuna. Un caso paradigmático son las regiones semiáridas, localizadas en los límites de las áreas de influencia de climas húmedos y secos, como una transición entre ambos tipos. Son regiones altamente vulnerables justamente porque su posición de "frontera" climática entre dominios contrastados las convierte en extremamente irregulares. Fases alternadas de lluvias benéficas y largos períodos secos son su principal seña de identidad. Estas características siempre han llamado la atención de los investigadores, son bien conocidos y estudiados en la literatura científica y ocupan espacios destacados en los manuales de Climatología más célebres. Dentro del dominio de los Climas semiáridos son particularmente conocidos el Sahel Africano y el Sertão del Nordeste del Brasil (a modo de ejemplo de sus características climáticas, véase la Figura 1), que han hecho correr auténticos ríos de tinta mediática, pero también científica y académica.

Muchas veces, las noticias nos hablan acerca de los episodios de sequía y sus consecuencias devastadoras sobre la actividad agropecuaria y la misma población humana, tal como está ocurriendo precisamente ahora, en los últimos años (desde 2011 hasta ahora) en el Sertão nordestino, que está padeciendo una de las peores sequías del último medio siglo. Siempre que esto sucede, todo parece indicar que la sequía es la única culpable de los gravísimos problemas sociales que allí tienen lugar, en una suerte de Determinismo Ambiental que liga de manera fatalista el destino de sus habitantes a los azares climáticos (Winter, 1999). Sin embargo, muchos autores y nosotros mismos creemos que los desastres no son causados solamente por la sequía, por el sencillo motivo de que ésta tiene lugar con frecuencia y los habitantes de estas regiones las conocen bien y saben cómo convivir con ello, y sí por la mala gestión de los recursos naturales (Elias, 1992; Alves, 2008), y los enquistados problemas de acceso a la tierra y extrema desigualdad social (con una oligarquía terrateniente con enorme fuerza política) que caracterizan de siempre esta extensa región. Dicho con otras palabras, los habitantes de esta región de Brasil han adoptado, por una parte, una actitud de Resiliencia bastante notable, concepto propio de la Psicología sobre el que no existe una definición universalmente aceptada pero que podemos considerar como "...competencia o un positivo y efectivo afrontamiento en respuesta al riesgo y a la adversidad" (Becoña, 2006: 127), o lo que es lo mismo, la capacidad para responder satisfactoriamente a circunstancias adversas de la vida, en este caso las duras condiciones climáticas. Y por otra parte, no se pueden dejar de lado serios problemas sociales de fondo arraigados desde hace mucho tiempo, manifestados en profundas desigualdades en el acceso a la tierra y al agua cuya importancia es decisiva para entender muchos problemas en tiempos de escasez. Diversos autores han denunciado en repetidas ocasiones este serio problema estructural, que está en la raíz misma de la miseria de gran parte de su población, más allá de las dificultades impuestas por el Clima (Alves, 2008). No obstante, sí hay que admitir que el inadecuado manejo del medio natural empeora aún más la situación. Por ejemplo, Jean Demangeot en su célebre obra "Los medios "naturales" del globo", alude 
a estos ambientes semiáridos señalando que serían perfectamente habitables si fuesen adecuadamente tratados, cosa que infelizmente no sucede (Demangeot, 1989).

Antes de continuar, conviene detenerse en este punto para aclarar qué entendemos por Sertão, pues es un concepto que en Brasil es manejado con mucha frecuencia y aunque desde fuera puede parecer que hace exclusiva referencia al semiárido nordestino, en realidad no es así. Según Filho (2011), el término Sertão tiene origen en los colonizadores portugueses y hace referencia, entonces como ahora, a espacios interiores, lejanos, apartados, no necesariamente secos. Es una noción de lejanía, normal en un país tan extenso como Brasil con amplios espacios interiores poco poblados. Eso sí, conviene distinguir de qué Sertão se está hablando, y es por ello que Filho (op. cit.) señala que Ab’Saber, uno de los más ilustres geógrafos brasileños, siempre hizo hincapié en este importante detalle. De ahí que en este texto hablemos expresamente de Sertão del Nordeste de Brasil, pues es específicamente el que estudiamos y no otros.

\section{LOS MECANISMOS REGULADORES DEL CLIMA EN EL NORDESTE DE BRASIL Y SU PREDICCIÓN}

Por lo tanto, la adaptación a las condiciones ambientales es clave para evitar o al menos reducir los impactos negativos. Y en el mundo de hoy es también crucial (y técnicamente viable) anticipar eventos y planificar actividades con base en previsiones meteorológicas de medio y largo plazo. El Sertão nordestino (Fig. 2) es un área particularmente atrayente para los investigadores por dos razones importantes: las lluvias irregulares, ya referidas, y su status como un clima "extraño", pues ni su posición latitudinal ni el efecto de abrigo orográfico pueden explicar por sí solos su existencia ${ }^{2}$. Las razones fundamentales hay que buscarlas en la circulación general atmosférica y sus relaciones con la temperatura de los océanos tropicales próximos (Atlántico y Pacífico). Precisamente en esta vía de investigación varios autores han trabajado en profundidad desde hace mucho tiempo.

Figura 1. Diagrama Ombrotérmico de Paulo Afonso (Bahia-Brasil). Período 1961-1990

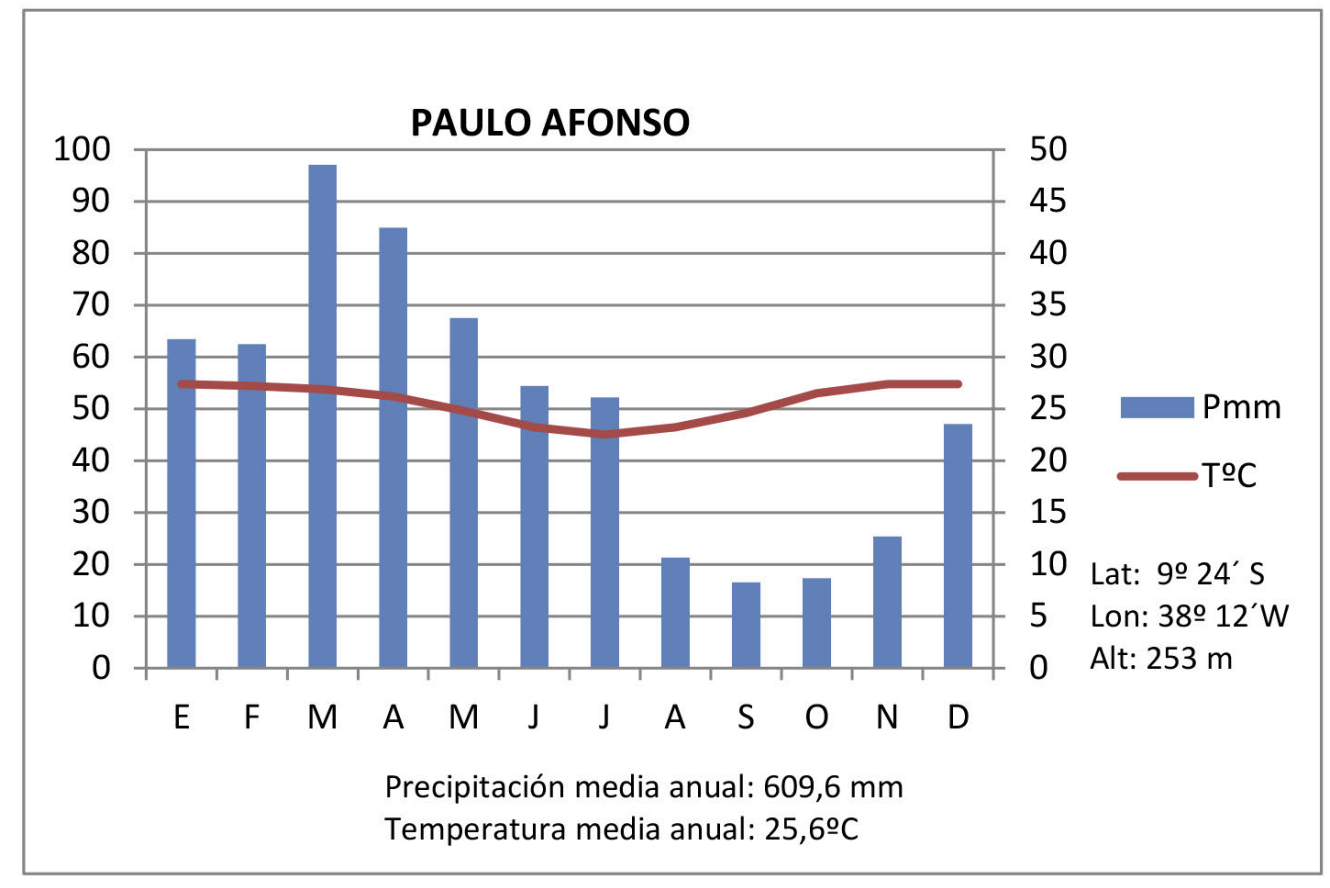

Fuente: Instituto Nacional de Meteorologia (INMET), Brasil. Elaboración propia.

1 Tradicionalmente, el criterio para delimitar el Semiárido en Brasil es la isoyeta de $800 \mathrm{~mm}$ anuales de precipitación. El 10 de Marzo de 2005, el Ministério da Integração Nacional mantuvo el criterio pluviométrico y añadió otros dos: Índice de Aridez de hasta 0,5 calculado por el balance hídrico del período 1961-1990, y Riesgo de Sequia mayor del 60\% tomando como base el período 1970-1990. Con estos criterios, el área Semiárida asciende a $982.563 \mathrm{Km}^{2}$ (SENA, 2007). A modo de comprobación, véase la Figura 2.

2 Ciertos enclaves del Sertão nordestino si padecen el efecto de barrera orográfica. Uno de los más conocidos es el área de los Cariris, a sotavento de la Sierra de Borborema (Estado de Ceará), pero son casos concretos dentro de un área semiárida muy extensa. 
Podemos citar, entre otros, los trabajos de Nobre y Shukla (1996), Baldicero y Oliveira (2000), Haylock et al.(2006), Andreoli y Kayano (2007), Kucharski et al.(2008), Nascimento y Palmeira (2008), o Beserra et al.(2011), que observan el problema de las teleconexiones atmosfera-océano desde una perspectiva general para el Nordeste brasileño en conjunto, la misma sobre la que indagan Costa y Barbosa (2007) aportando estudios sobre los efectos en la vegetación. También son numerosos los trabajos que estudian este mismo asunto, si bien centrándose en una determinada región, como el ejemplo de Costa et al.(2009) para el Estado de Ceará. Otra perspectiva analiza la relación de la variabilidad pluviométrica con el régimen de vientos, tema estudiado por Cavalcante y Alonso (2006).

En todos estos trabajos, si dejamos de lado pequeñas diferencias de matiz, podemos observar una unanimidad casi total en explicar muchas de las variaciones interanuales de precipitación en el Sertão nordestino en base a las influencias de las anomalías de temperatura de las aguas del Pacífico y Atlántico tropicales, esto es, los fenómenos El Niño-La Niña y el llamado Dipolo del Atlántico. Conviene, sin embargo, hacer una importante anotación. Las explicaciones clásicas ligaban los períodos de sequia en el Nordeste de Brasil a la ocurrencia concomitante del fenómeno El Niño, pero muchos de los estudios consultados para este trabajo, antes reseñados, ponen en evidencia que tal relación no es tan concluyente como se suponía, o al menos no lo es en todos los casos. Más importancia tiene, en cambio, la temperatura del Atlántico Tropical, como descubren Nobre y Shukla (1996) o Andreoli y Kayano (2007). Aquí sí parece existir un vínculo más claro con las precipitaciones, lo que no deja de ser lógico por una simple cuestión de proximidad: el Atlántico baña directamente el Litoral Nordeste de Brasil.

Figura 2. Delimitación y localización del Semiárido en Brasil

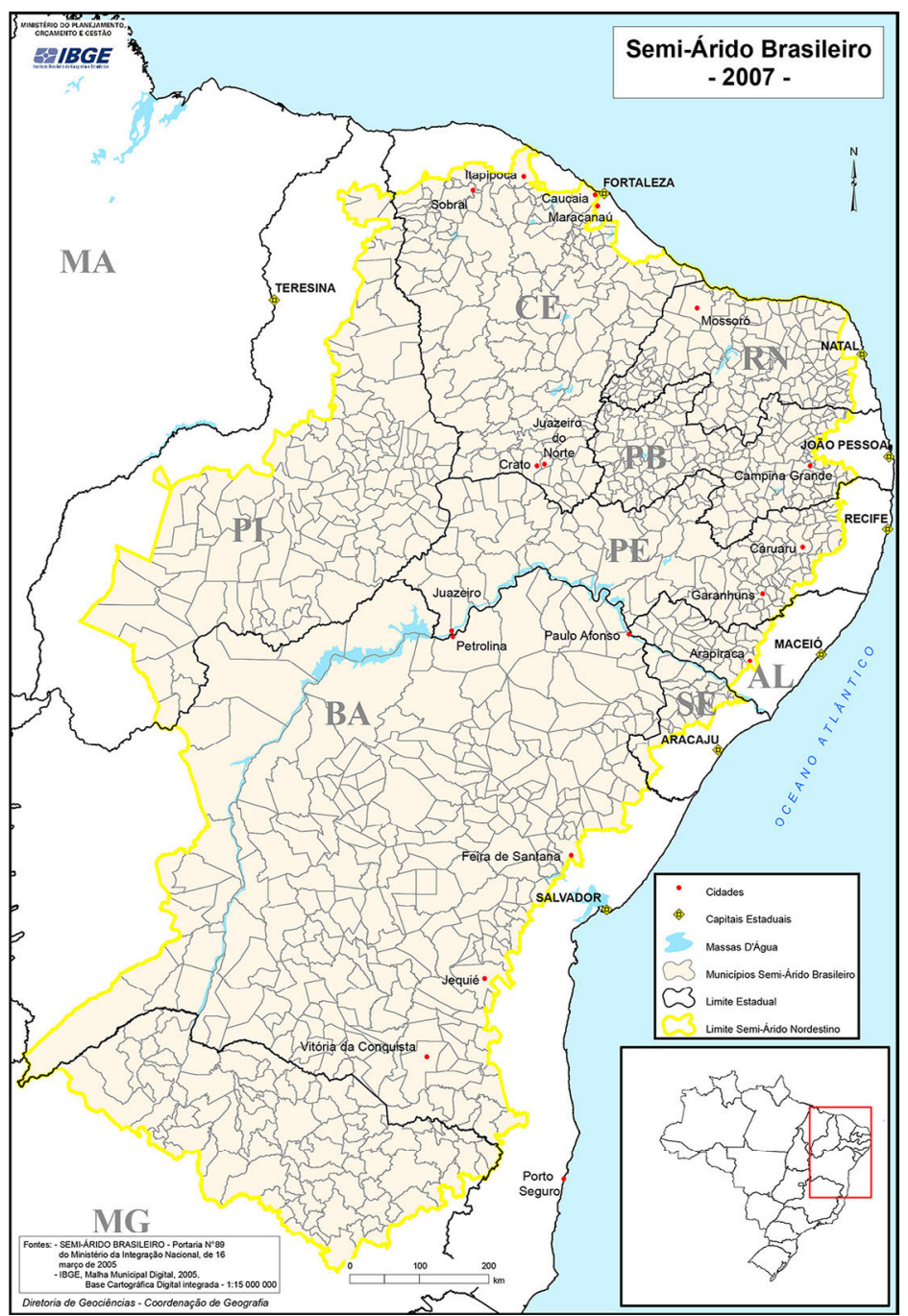

Fuente: Instituto Brasileiro de Geografia e Estatistica (IBGE). Localización de Paulo Afonso incluida por el autor. 
Estos procesos pueden ser detectados con cierta antelación gracias a la continua medición de la temperatura oceánica a través de satélites y buques de investigación, y conociendo el comportamiento habitual de la atmosfera cuando estas oscilaciones de la temperatura oceánica tienen lugar, es posible hacer una previsión razonablemente aproximada sobre las condiciones meteorológicas en los próximos meses, en particular si la precipitación tenderá a ser mayor o menor de las medias climáticas. Sin embargo, las previsiones de este tipo poseen un margen de error que no conviene despreciar, y prueba de esto son trabajos tan ilustrativos como el de Brabo et al.(2007) en el que se manifiesta una cierta discrepancia en los resultados de modelos de previsión estacional estadístico-dinámica de la precipitación del Nordeste del Brasil. Además, estas previsiones son probabilísticas, es decir, indican una probabilidad de ocurrencia mayor o menor de determinado evento (en este caso, el porcentaje de probabilidades de tener lluvias por encima o por debajo de la media), no indican certezas, y este hecho, como veremos más tarde, lleva a lamentables confusiones y desilusiones entre los usuarios finales de esta información, como pueden ser los agricultores. Entre tanto, no hay duda de que esta información es una inestimable ayuda para la planificación de actividades agrícolas y pecuarias, tanto en términos de estimar las etapas de siembra más adecuadas como una estimación de la cosecha final y las necesidades de irrigación y de alimento para el ganado. La importancia de este tema queda clara en trabajos como los de Azevedo y Santos (2007) o Brabo et al.(2008), que prestan atención a los riesgos climáticos para determinados cultivos (algodón) y sobre la sostenibilidad agrohidrometeorológica, teniendo en cuenta la irregularidad de las precipitaciones.

Es por tanto la irregularidad pluviométrica una de las principales señas de identidad del Clima existente en el Semiárido del Nordeste de Brasil, característica que dificulta todavía más la misión de predecir con antelación cómo van a ser las lluvias y eventualmente pone en serios compromisos a los profesionales meteorólogos, que quedan en entredicho si sus previsiones fallan. Es tarea delicada y al mismo tiempo imprescindible informar a la sociedad de las previsiones meteorológicas, y más aún a los agricultores, directamente afectados por los vaivenes atmosféricos. Es precisamente la dificultad de comunicar y hacer entender las previsiones a los pequeños productores la parte central de este trabajo, que pasamos a analizar en el apartado siguiente.

\section{LAS DIFICULTADES DE TRANSMITIR PREVISIONES METEOROLÓGICAS ESTACIONALES A PEQUEÑOS AGRICULTORES: ENFOQUES TEÓRICO-PRÁCTICOS Y POSIBILIDADES DE APLICACIÓN EN EL SERTÃO NORDESTINO}

Como hemos visto en el apartado previo, existe la posibilidad de conocer con un cierto margen temporal cómo se comportarán las lluvias en el Sertão, teniendo en cuenta la conexión entre las lluvias y el comportamiento térmico de las aguas oceánicas adyacentes. Pero es de la máxima importancia que esta información llegue a todos los interesados, y en particular a los pequeños agricultores que, por un lado, suelen estar más expuestos a los eventos climáticos extremos y, por otro lado, tienen una capacidad de recepción de la información muy limitada. Es pues pertinente saber cómo llega la información meteorológica a estas personas y si es adecuada a sus intereses.

En Brasil existe una previsión trimestral "de consenso" de las condiciones climáticas básicas (precipitación y temperatura) elaborada de común acuerdo por el Instituto Nacional de Meteorologia (INMET) y el Centro de Predição do Tempo e Estudos Climáticos del Instituto Nacional de Pesquisas Espaciais (INPE-CPTEC). Este pronóstico es difundido en las páginas web de estos organismos en forma de Boletín (Fig. 3), por los medios de comunicación (menos detallado), y también por otros organismos como el Instituto de Médio Ambiente e Recursos Hídricos (INEMA), órgano perteneciente al gobierno del Estado de Bahía, que junto con el pronóstico general para Brasil introduce anotaciones específicas para el estado. Esta previsión toma en consideración múltiples factores atmosféricos y oceánicos de los que se ha hablado anteriormente, y establece la probabilidad de que las precipitaciones y las temperaturas sean superiores o inferiores a las normales climáticas. El pronóstico es entonces publicado como X porcentaje de probabilidad de ocurrencia. Este método de pronóstico estacional es habitual en las principales agencias meteorológicas del Mundo. El potencial de estos pronósticos para la planificación de actividades agrícolas y pecuarias es muy importante, y realmente una de las razones de su elaboración es precisamente ayudar en este tipo de acciones. Sin embargo, la transmisión de este tipo de información en concreto a pequeños agricultores es una misión de gran complejidad que en el ámbito académico ha encontrado un amplio tratamiento.

Algunos trabajos centran su atención en la perspectiva teórica del asunto, visto como un complejo proceso de comunicación (Taddei, 2008; Weiss et al. 2000; Rijks et al. 2000; Blench, 1999; Mukhala, 2000), 
y otros trabajos, entre tanto, analizan casos concretos partiendo del mismo marco teórico-conceptual en espacios semiáridos como Mali (Hellmuth et al., Zimbabwe (Pitt et al. 2002), Burkina Faso (Ingram et al. 2002), y el Estado de Ceará en Brasil (Lemos et al. 2002; Pennesi, 2007).

Figura 3. Previsión climática "de consenso" de las precipitaciones correspondientes al Trimestre Julio-Agosto-Septiembre de 2013

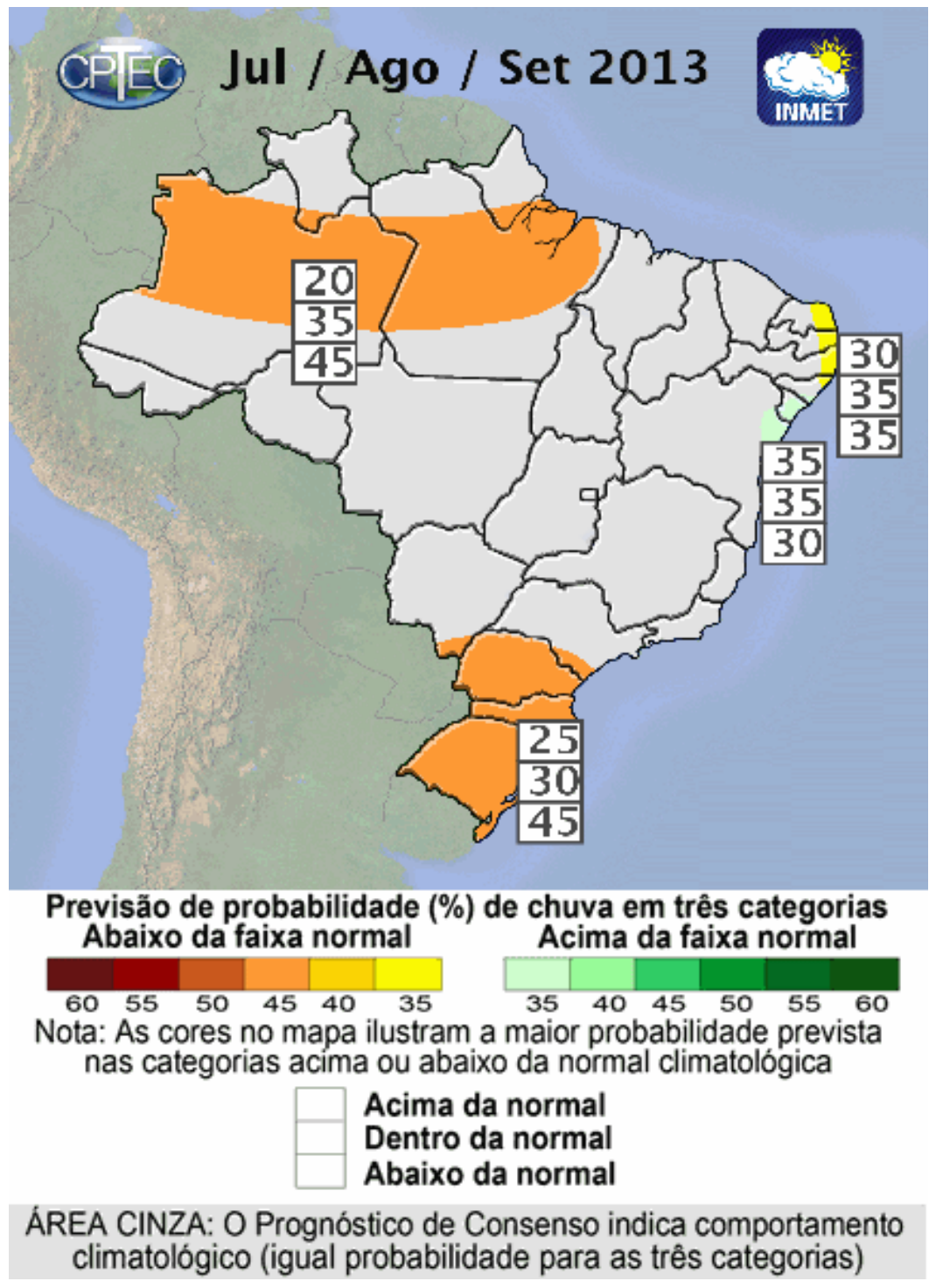

Fuente: Centro de Previsão do Tempo e Estudos Climáticos (CPTEC), Instituto Nacional de Pesquisas Espaciais (INPE), Brasil.

El magnífico trabajo de Taddei (2008) recoge perfectamente las bases conceptuales de esta problemática. Este autor señala que el proceso de comunicar la información meteorológica a pequeños agricultores suele ser muy poco efectivo por una razón simple: el mensaje no está adaptado al receptor final. Dicho con otras palabras: los agricultores no comprenden la información porque los servicios meteorológicos y los medios de comunicación lo transmiten en un lenguaje técnico (en el caso de los medios, salpimentado por ciertas dosis de sensacionalismo) que lo transforma en inútil e irrelevante para el destinatario final. A esta dificultad básica se unen otras como la imposibilidad de que las previsiones alcancen una escala local (el agricultor se interesa, como es lógico, por lo que pueda suceder con las lluvias en su pueblo o comarca, no en la totalidad de la región, y los modelos de predicción no tienen ese nivel de detalle), y la difícil comprensión del carácter probabilístico de la previsión (como dijimos antes, indica probabilidades y no certezas, por lo que existe un margen de error). Así, es frecuente que cuando una previsión indica que los próximos meses habrá X porcentaje de probabilidades de que las lluvias sean mayores o menores 
de lo "normal", los agricultores perciben esto como dato exacto, de modo que si finalmente sucede lo contrario de lo indicado por el pronóstico (especialmente si se produce sequía habiendo previsión de lluvias generosas) las críticas son duras y se produce una notable pérdida de confianza y de credibilidad en la fuente informativa y en el organismo que elabora y difunde el pronóstico. El mismo concepto de "normal" resulta equivoco para gran parte de los agricultores, pues toma en consideración el volumen total de lluvias y no considera el reparto mensual de las lluvias (regularidad) y si habrá períodos secos intercalados durante la estación lluviosa (los llamados veranicos en el Sertão), información que si interesa, y mucho, a los agricultores, pues puede comprometer la cosecha mucho más que las cantidades totales de lluvia. Este asunto es altamente delicado en un contexto en el que la agricultura familiar de subsistencia, aún muy poco evolucionada técnicamente, depende de unas lluvias escasas e irregulares para poder dar sustento a gran número de personas. La Seca es parte fundamental de la cultura Sertaneja, de las condiciones de la naturaleza con las que la gente lucha y a la vez convive desde hace muchas generaciones, y el estado de "ansiedad" por la llegada o no de las lluvias en el momento adecuado siempre está presente en el ánimo de la colectividad. Así, es habitual en el Sertão nordestino (y en la totalidad de los espacios con condiciones climáticas similares) que el agricultor no arriesgue su cosecha por unos pronósticos con alto grado de incertidumbre y adopte una estrategia de cultivo en la que mezcle variedades adaptadas a humedad y a sequedad, aún a condición de tener que replantar si la lluvia tarda en llegar (...estão minimizando a perda de oportunidade da chuva, ainda que desperdiçando sementes. Isso é compreensível, dado o fato de que sementes estão disponíveis no mercado e chuva não está. TADDEI, 2008, P.82).

Ni siquiera fían toda su suerte a los llamados "Profetas da Chuva", agricultores que pronostican las condiciones de la estación lluviosa observando las señales de la naturaleza, un saber transmitido de padres a hijos: comportamiento de los animales, desarrollo de las plantas o elementos astronómicos, combinado con profunda Fe religiosa (Folhes et al. 2007). Estas personas gozan de una elevada credibilidad en el Sertão, fundamentalmente porque también son agricultores y "conectan" directamente con los conocimientos y mentalidades del mundo rural (una interesante reseña de la transformación de los "Profetas da Chuva" en fenómeno mediático inserido en el imaginario "urbano" del folclore Sertanejo puede encontrarse en Taddei, 2009).

Por lo tanto, existe un importante problema de comunicación en el que el mensaje transmitido es inadecuado para el receptor, y este hecho compromete seriamente la credibilidad de la información y de la propia fuente. En este contexto, es de una importancia transcendental adquirir un conocimiento lo más detallado posible de las demandas informativas del receptor final, lo que pasa por conocer su mentalidad y sensibilidad, no menospreciar su experiencia y conocimientos acumulados sobre el medio natural (frecuentemente el agricultor opina que existe una falta de respeto de los conocimientos tradicionales por parte de los organismos que elaboran los pronósticos, gente ajena al Sertão que no conoce su realidad como ellos), para poder alcanzar al mayor número posible de personas con un mensaje claro y comprensible.

Un magnífico ejemplo de los peligros que acechan tras la falta de entendimiento entre organismos emisores de pronósticos y agricultores es lo que sucedió con la Fundação Cearense de Meteorologia e Recursos Hídricos (FUNCEME) en el Estado de Ceará (vid. Lemos et al. 2002 y Pennesi, 2007). Esta unidad de la federación brasileña fue pionera en la utilización de pronósticos estacionales en la planificación agrícola, pues desde los años 90 del pasado siglo la FUNCEME elabora y difunde esta información tanto para gestores públicos involucrados en el desarrollo agrario y la gestión de recursos hídricos como para agricultores. Después de una inicial etapa de ilusión y entusiasmo, el modelo no tardó mucho tiempo en quebrar por los problemas señalados anteriormente: lenguaje inadecuado, pronóstico probabilístico mal entendido, escala espacio-temporal poco útil. Los primeros pronósticos "equivocados" derivaron en fuertes disputas y tensiones sociales y políticas (hemos explicado antes que el pronóstico probabilístico no contempla la regularidad de las lluvias, solamente la cantidad total) y en un descrédito importante de la FUNCEME, de manera que los agricultores no confían más en estos pronósticos, que consideran desvinculados de la realidad natural (los "Profetas da Chuva", en este contexto, alcanzan especial resonancia en Ceará, vid. Taddei, 2009 y Folhes et al. 2007).

Los casos prácticos que hemos consultado para este trabajo (Mali, Burkina Faso y Zimbabwe) incluyen reuniones y entrevistas personales con pequeños agricultores con el objetivo de saber directamente si la información meteorológica llega hasta ellos y de qué manera, así como sus demandas de información, y también para explicarles con claridad qué es y qué supone un pronóstico probabilístico y en última instancia, lo que significan en realidad. Los resultados en todos los casos son los mismos: la 
información llega a través de los medios de comunicación (la radio tiene un papel clave por causa de las altas tasas de analfabetismo existente en estas regiones y por su amplia cobertura espacial) y por medio de las agencias de desarrollo agrario, con un lenguaje y unos contenidos inadecuados y/o poco útiles para sus actividades. Salen a la luz los problemas de escala de la información (es técnicamente imposible proporcionar una información de detalle a escala local, pues los modelos no analizan a ese nivel) y de la regularidad de las lluvias. Los agricultores consideran de la mayor importancia para obtener una cosecha abundante saber cuándo comenzará la lluvia (incluidas las lluvias precoces de "pre-estación"), cuánto van a durar y si caerán con regularidad para poder planificar los cultivos y sus ciclos, informaciones éstas que también están fuera del alcance de los modelos de previsión a día de hoy. El problema de la comprensión del carácter probabilístico de la previsión, siempre señalado como uno de los más difíciles de solucionar, es explicado utilizando ejemplos tomados de los juegos de azar, como una caja con 100 papeles de tres colores en proporción a las probabilidades indicadas por el pronóstico (es el caso de Mali estudiado por Hellmuth et al., experimento que permite a los agricultores comprobar que en estos pronósticos, la mayor probabilidad de una estación con lluvias abundantes no significa que no exista posibilidad alguna de que al final sea muy seca (en un caso como ese, las probabilidades de sacar el papel que indica año seco son pequeñas por el hecho de haber menor número de papeles de ese color, pero no inexistentes).

\section{REFLEXIONES FINALES}

Las previsiones meteorológicas estacionales, sin ser una ciencia exacta, son una gran ayuda a la hora de planificar actividades agrícolas y ganaderas, pues permiten obtener una visión aproximada de cómo se comportará la atmósfera en el futuro inmediato. Pero esa información debe ser tratada con sumo cuidado para no dar lugar a confusiones, en primer lugar, y en segundo lugar, tiene que ser difundida de manera tal que llegue a los directamente interesados de forma fácil y que sea portadora de un mensaje comprensible. De lo contrario, lo que podría ser una potente herramienta que permita a los pequeños agricultores de áreas sensibles como las semiáridas manejar mejor sus limitados recursos, pasa a ser un elemento extraño y frustrante que empeora aún más la situación. En el caso específico del Sertão del Nordeste de Brasil, existe una previsión estacional bien elaborada que, sin embargo, aún no ha conseguido llegar al pequeño agricultor, y allá donde se ha intentado (recordemos el caso de Ceará) ha terminado mal por la falta de entendimiento entre las partes implicadas. Son éstas, por lo tanto, las cuestiones a tener presentes a la hora de aplicar esta valiosa información en el auxilio de los pequeños productores rurales. En el caso del Estado de Bahia, creemos viable aplicar la misma metodología propuesta por los autores aquí estudiados, y ello significa partir de un primer contacto personal con los agricultores aplicando un cuestionario previamente estructurado, combinando preguntas abiertas y cerradas sobre la información meteorológica a la que acceden, cómo acceden a ella, si la entienden, y cómo y qué desearían recibir, además de averiguar sus conocimientos sobre el Clima de la región en base a su experiencia vital. Partiendo de esta valiosa información, en un segundo momento es posible experimentar una nueva forma de transmisión de la información meteorológica, adaptada a sus necesidades y expectativas. Esa vía de comunicación tendría que ser consensuada a partir de las evidencias obtenidas del trabajo con los agricultores y testada con ellos mismos para verificar su funcionamiento. Caso de no funcionar satisfactoriamente, sería sustituída por otra hasta encontrar una que proporcione buenos resultados. En resumen, se persigue como objetivo prioritario proporcionar una información accesible al mayor número de productores posible, tanto desde el punto de vista físico como interpretativo, o dicho de otro modo, que la información meteorológica consiga llegar y que al mismo tiempo pueda ser entendida correctamente.

\section{REFERENCIAS BIBLIOGRÁFICAS}

ALVES, R. M. (2008): Entre o combate à seca e a convivência com o Semi-árido: transições paradigmáticas e sustentabilidade do desenvolvimento. Fortaleza, Banco do Nordeste (Teses e Dissertações, 12), 275 pp.

ANDREOLI, R.V.; KAYANO, M.T. (2007): "A importância relativa do Atlântico Tropical Sul e Pacífico Leste na variabilidade de precipitação do Nordeste do Brasil", en Revista Brasileira de Meteorologia, Vol.22, $\mathrm{n}^{\circ} 1$, pp. 63-74.

BALDICERO MOLION, L.C.; DE OLIVEIRA BERNARDO, S. (2000): "Dinâmica das chuvas no Nordeste brasileiro", en Anais do XI Congresso Brasileiro de Meteorologia, Rio de Janeiro. pp. 1334-1342.

BECOÑA, E.; (2006): "Resiliencia: definición, características y utilidad del concepto", en Revista de Psicopatología y Psicología Clínica, Vol. II, n³, pp. 125-146. 
BESERRA LUCENA, D.; GOMES FILHO, M.F; SERVAIN, J. (2011): “Avaliação do impacto de eventos climáticos extremos nos Oceanos Pacífico e Atlântico sobre a estação chuvosa no Nordeste do Brasil”, en Revista Brasileira de Meteorologia, Vol.26, n². pp. 297-312.

BLENCH, R. (1999): "Seasonal climate forecasting: Who can use it and how should it be disseminated?", en Natural Resource Perspectives, $\mathrm{n}^{\circ} 47$. Disponible en Internet (http://www.odi.org.uk/resources/ docs/2871.pdf). Acceso en 2-08-2012.

BRABO ALVES, J.M.; ARAÚJO COSTA, A.; SOUSA SOMBRA, S.; BEZERRA CAMPOS, J.N.; DE SOUZA FILHO, F. de A.; PASSOS RODRIGUES MARTINS, E.D.; DA SILVA, E. M.; SANTANA DOS SANTOS, A.C.; BARBOSA, H.; BARBOSA MELCIADES, W.L.; FERRAN MONCUNNIL, D. (2007): "Um estudo inter-comparativo de previsão sazonal estatística-dinâmica de precipitação no Nordeste do Brasil", en Revista Brasileira de Meteorologia, Vol.22, n³. pp. 354-372.

BRABO ALVES, J.M.; B. CAMPOS, J.N.; B. VIEIRA, V. de P. (2008): "Análise de sustentabilidade agrohidrometeorologica no estado do Ceará", en Revista Brasileira de Meteorologia, Vol.23, n.1. pp. 103-114.

CAVALCANTE DE LIMA MOSCATI, M.; ALONSO GAN, M. (2006): "Rainfall variability in the rainy season of semiarid zone of Northeast Brazil (NEB) and its relations to wind regime", en International Journal of Climatology, $\mathrm{n}^{\circ} .27$, pp. 493-512.

CAVAlCANTI, I.F.A. (2009): Tempo e Clima no Brasil. São Paulo, Oficina de Textos. 463 pp.

COSTA DOS SANTOS, C.A.; BARBOSA DE BRITO, J.I. (2007): "Análise dos índices de extremos para o semi-árido do Brasil e suas relações com TSM e IVDN", en Revista Brasileira de Meteorologia, Vol.22, $\mathrm{n}^{\circ} .3$, pp. 303-312.

COSTA DOS SANTOS, C.A.; BARBOSA DE BRITO, J.I.; RAMANA RAO, T.V.; ALENCAR MENEZES, H.E. (2009): "Tendências dos índices de precipitação no estado do Ceará", en Revista Brasileira de Meteorologia, Vol.24, $\mathrm{n}^{\circ}$.1. pp. 39-47.

DEMANGEOT, J. (1989): Los medios “naturales” del globo. Barcelona, Masson. 251 pp.

ELIAS DE CASTRO, I. (1992): O mito da necessidade: discurso e prática do regionalismo nordestino. Rio de Janeiro, Bertrand do Brasil, 247 pp.

FILHO, F. D. A. (2011): "Sobre a palavra "Sertão": origens, significados e usos no Brasil (do ponto de vista da ciência geográfica)", en Ciência Geográfica, Vol. XV (1), pp. 84-87.

FOLHES, M.T.; DONALD, N. (2007): "Previsões tradicionais de tempo e clima no Ceará: o conhecimento popular à serviço da ciência", em Sociedade e Natureza, n.19 (2). pp. 19-31.

HAYLOCK, M.R.; PETERSON, T.C.; ALVES, L.M.; AMBRIZZI, T.; ANUNCIAÇÃO, Y.M.T; BAEZ, J.; BARROS, V.R.; BERLATO, M.A.; BIDEGAIN, M.; CORONEL, G.; CORRADI, V.; GARCIA, V.J.; GRIMM, A.M.; KAROLY, D.; MARENGO, J.A.; MARINO. M.B.; MONCUNILL, D.F; NECHET, D.; QUINTANA, J.; REBELLO, E.; RUSTICUCCI, M.; SANTOS, J.L.; TREBEJO, I.; VINCENT, L.A. (2006): "Trends in total and extreme South American rainfall in 1960-2000 and links with Sea Surface Temperature", en Journal of Climate, Vol.19, pp. 1490-1512.

HELLMUTH, M.; DIARRA, D.Z.; VAUGHAN, C.; COUSIN, R.: "Increasing food security with agrometeorological information: Mali's National Meteorological Service helps farmers manage climate risk", en World Resources Report. Disponible en Internet (http://www.wri.org/sites/default/ files/wrr case study increasing food security mali .pdf). Acceso en 2-08-2012.

INGRAM, K.T.; RONCOLI, M.C.; KIRSHEN, P.H. (2002): "Opportunities and constraints for farmers of West Africa to use seasonal precipitation forecast with Burkina Faso as a case study", en Agricultural Systems, no74, pp. 331-349.

KUCHARSKI, F; POLZIN, D.; HASTENRATH, S. (2008): "Teleconnection mechanisms of Northeast Brazil droughts: modeling and empirical evidence", en Revista Brasileira de Meteorologia, Vol.23, n², pp. 115-125.

LEMOS, M.C.; FINAN, T.J.; FOX, R.W.; NELSON, D.R.; TUCKER, J. (2002): "The use of seasonal climate forecasting in policymaking: lessons from Northeast Brazil", en Climate Change, n55, pp. 479-507

MARENGO, J. A. (2008): Vulnerabilidade, impactos e adaptação à mudança do clima no semi-árido do Brasil. Brasília, Parcerias Estratégicas.

MUKHALA, E. (2000): "Meteorological services and farmers in Africa: Is there shared meaning?", en Sustainable Development Department, FAO.

NASCIMENTO BEZERRA, A.C.; PALMEIRA CAVALCANTI, E. (2008): "Energia estática sobre o Norte e Nordeste do Brasil relacionada com a temperatura da superfície do mar", en Revista Brasileira de Meteorologia, Vol.23, n², pp. 239-263. 
NOBRE, P.; SHUKLA, J. (1996): "Variations of Sea Surface Temperature, Wind Stress and Rainfall over the Tropical Atlantic and South America", en Journal of Climate, Vol.9, pp. 2464-2479.

PATT, A.; GWATA, C. (2002): "Effective seasonal climate forecast applications: examining constraints for subsistence farmers in Zimbabwe", en Global Environment Change, $\mathrm{n}^{\circ} 12$, pp. 185-195.

PENNESI, K. (2007): "Improving forecast communication. Linguistic and cultural considerations", en Bulletin of American Meteorological Society, Vol.88, nº7, pp. 1033-1044.

RIJKS, D.; BARADAS, M.W. (2000): "The clients for agrometeorological information", en Agricultural and Forest Meteorology, $\mathrm{n}^{\circ} 103$, pp. 27-42.

SENA PEREIRA JR., J. (2007): Nova delimitação do Semi-árido brasileiro, Consultoría Legislativa, Câmara dos Deputados, Brasília. 24 pp.

TADDEI, R. (2008): "A comunicação social de informações sobre tempo e clima: o ponto de vista do usuário", en Boletim do SBMET Agosto-Diciembre 2008. pp. 76-86.

TADDEI, R. (2009): "Os profetas da chuva do Sertão como produção midiática", Trabajo presentado para la reunión anual de 2009 de la Latin America Studies Association. Disponible en Internet (http://lasa. international.pitt.edu/members/congress-papers/lasa2009/files/TaddeuRenzo.pdf) Acceso en 2-08-2012.

VIEIRA DE AZEVEDO, P.; DOS SANTOS SILVA, F.D. (2007): "Risco climático para o cultivo do algodoeiro na região Nordeste do Brasil”, en Revista Brasileira de Meteorologia, Vol.22, nº.3. pp. 408-416.

VVAA (1998): Análise dos atributos climáticos do Estado da Bahia. Salvador, Superintendência de Estudos Econômicos e Sociais da Bahia. 85 pp.

WEISS, A.; VAN CROWDER, L.; BERNARDI, M. (2000): "Communicating agrometeorological information to farming communities", en Agricultural and Forest Meteorology, nº103, pp. 185-196.

WINTER RIBEIRO, R. (1999): "Seca e determinismo: a gênese do discurso do Semi-Árido nordestino", em Anuário do Instituto de Geociências - UFRJ, Vol. 22, pp. 60-91. 